\title{
Convergence of Product Integration Rules for Functions With Interior and Endpoint Singularities Over Bounded and Unbounded Intervals
}

\author{
By D. S. Lubinsky* and Avram Sidi
}

\begin{abstract}
The convergence of product integration rules, based on Gaussian quadrature points, is investigated for functions with interior and endpoint singularities over bounded and unbounded intervals. The investigation is based on a new convergence result for Lagrangian interpolation and Gaussian quadrature of singular integrands.
\end{abstract}

1. Introduction. The standard product integration rule has the form

$$
\int_{a}^{b} f(x) k(x) d x \simeq \sum_{i=1}^{n} w_{n i} f\left(x_{n i}\right)
$$

where $k(x)$ is a badly behaved function; the $\left\{x_{n i}\right\}$ are chosen in advance of the $\left\{w_{n i}\right\}$, while the $\left\{w_{n i}\right\}=\left\{w_{n i}(k)\right\}$ are determined so as to make the rule exact for a class of functions, such as the polynomials of degree less than $n$. A particularly attractive choice of the $\left\{x_{n i}\right\}$ is the zeros of orthogonal polynomials. For this case, a detailed analysis was carried out by Sloan and Smith [13, and references therein]. They proved convergence for the case where $(a, b)$ is bounded; $f(x)$ is bounded and Riemann integrable; the orthogonal polynomials correspond to a weight function $w(x)=\alpha^{\prime}(x)$ positive almost everywhere in $(a, b)$; and $k(x)$ satisfies a weak integrability condition. More recently, Smith, Sloan and Opie [14] considered infinite intervals with product integration rules based on the Gauss-Hermite and GaussLaguerre points.

The principal contribution of this paper is to show that product integration rules based on Gauss quadrature points still converge when the function $f(x)$ has finitely many interior and endpoint singularities. Such a study may at first seem counter to the spirit of product integration, for usually $k(x)$ is singular and $f(x)$ is smooth. However, there are cases-for example, in the solution of certain integral equations - where $f(x)$ is not smooth, and the locations of its singularities are unknown, or difficult to estimate, so they cannot be absorbed into $k(x)$. Cognizance of this fact was taken by Rabinowitz and Sloan [11] who were the first to prove convergence of product integration rules for singular integrands. Their results were primarily for

Received March 23, 1984; revised January 15, 1985.

1980 Mathematics Subject Classification. Primary 41A55; Secondary 65D30.

Key words and phrases. Gauss quadrature, Lagrange interpolation, product integration rules, convergence, singular integrands.

* Research completed while author was Lady Davis fellow at Technion, Haifa, Israel. 
piecewise polynomial quadrature rules, but they included some general convergence results [11, Theorem 1, Corollaries 1 and 2]. However, our results are not included in theirs, because their condition (18) in Corollary 1 [11] is difficult to verify, and not true for some of the weight functions and kernels $k(x)$ considered here. This is so, because the weights $w_{n i}$ in the product integration rules based on Gauss quadrature points involve the partial sums $S_{n}[k]\left(x_{n i}\right)$ of the orthonormal expansion of $k(x)$, and the latter may be unbounded even when $k(x)$ satisfies the integrability conditions used in this paper.

A second feature of the main result (Theorem 4.2) is that the weight function $d \alpha(x)$ is restricted only in the sense that it must be the unique solution of its moment problem. Hence $d \alpha(x)$ can be a pure jump distribution, and the interval $(a, b)$ containing its support may be unbounded. Unfortunately, the generality of the weight function sacrifices some generality of the kernel $k(x)(d \beta / d \alpha)(x) \alpha^{\prime}(x)$ in our notation), so that the conditions on the kernel involve $(k)^{2}$, rather than $|k|^{p}$ for some $p>1$. The reason for this is that mean convergence in $L_{p}$ of Lagrangian interpolation at zeros of orthogonal polynomials holds for general weights only when $p=2$ (Nevai [8]).

Along the way to proving Theorem 4.2, we establish a new result on convergence of Gaussian quadrature and $L_{2}$ convergence of Lagrange interpolation for functions with interior singularities, i.e., Theorem 3.5. This result is the corrected version of Problems 12 and 13 in [4, pp. 132-133] and is more general than any previous result for Gaussian quadrature of singular integrands.

2. Notation. (i) Throughout, $(a, b)$ will be a fixed real interval $(-\infty \leqslant a<b \leqslant \infty)$ and $\alpha:(a, b) \rightarrow \mathbf{R}$ will be right continuous, monotone increasing with infinitely many points of increase such that

$$
\alpha_{j}=\int_{a}^{b} x^{j} d \alpha(x)<\infty, \quad j=0,1,2, \ldots
$$

We assume that, apart from normalization, $\alpha$ is the unique solution of the (Hamburger) moment problem (2.1) - see for example Freud [4, Chapter 2].

(ii) $\varphi_{0}, \varphi_{1}, \varphi_{2}, \ldots$ will be the sequence of orthonormal polynomials for $\alpha$, and $a<x_{n 1}<x_{n 2}<\cdots<x_{n n}<b$ will denote the zeros of $\varphi_{n}$, while we write $x_{n 0}=a$, $x_{n, n+1}=b, n=1,2,3, \ldots$. The Gauss-Jacobi quadrature rule of order $n$ is

$$
\int_{a}^{b} g(x) d \alpha(x) \simeq K_{n}(g)=\sum_{j=1}^{n} \lambda_{n j} g\left(x_{n j}\right)
$$

and is exact for polynomials of degree less than $2 n$. When $g(x)$ has finitely many singularities in $(a, b)$, we modify (2.2) as follows: Suppose for some positive integer $l$

$$
\left\{\begin{array}{l}
a<y_{1}<y_{2}<\cdots<y_{l}<b \text { are those points in }(a, b) \\
\text { for which } \lim \sup _{x \rightarrow y_{i}}|g(x)|=\infty, i=1,2, \ldots, l .
\end{array}\right.
$$

Define $\tau(n, g)$ to be the subset of $\{1,2, \ldots, n\}$ such that $j \in \tau(n, g)$ if

$$
\text { either }\left\{y_{1}, y_{2}, \ldots, y_{l}\right\} \cap\left(x_{n, j-1}, x_{n, j+1}\right)=\varnothing
$$

$$
\left\{\begin{array}{l}
\text { or if for any } i, 1 \leqslant i \leqslant l, \text { such that } y_{i} \in\left(x_{n, j-1}, x_{n, j+1}\right) \\
\left|\alpha\left(x_{n j}\right)-\alpha\left(y_{i}\right)\right| \geqslant \lambda_{n j} .
\end{array}\right.
$$


Define

$$
K_{n}^{*}(g)=\sum_{j \in \tau(n, g)} \lambda_{n j} g\left(x_{n j}\right)
$$

The condition (2.4A) ensures that, in forming $K_{n}^{*}(g)$, we include those abscissas $x_{n j}$, which are not the closest among the $\left\{x_{n i}\right\}_{i}$ from the left or right, to any singularity of $g$. The condition (2.4B) ensures that we include also those abscissas $x_{n j}$ which are closest from the left or right to some singularity of $g$, provided they are not "too close" to the singularity.

One can modify (2.4B): $\lambda_{n j}$ can be replaced by $c \lambda_{n j}$ ( $c$ a positive constant). One can even omit (2.4B) altogether. In both these cases, the main results still hold, though one has then to modify Lemma 3.2 .

Note that $\tau(n, g)$ omits at most $2 l$ integers, that is at most two integers per interior singularity of $g$. When $g$ has no interior singularities, then $\tau(n, g)=$ $\{1,2, \ldots, n\}$ and $K_{n}^{*}(g)=K_{n}(g)$. We remark that when more is assumed about $\alpha(x)$, then $(2.4 \mathrm{~B})$ can be modified so that $\tau(n, g)$ omits at most one integer per interior singularity of $g$ (see [7]).

(iii) Whenever the (Lebesgue-Stieltjes) integrals are defined, we set

$$
S_{n}[g](x)=\sum_{j=0}^{n}\left\{\int_{a}^{b} g(t) \varphi_{j}(t) d \alpha(t)\right\} \varphi_{j}(x),
$$

that is, $S_{n}[g]$ is the $(n+1)$ st partial sum of the orthogonal series expansion of $g$ in the $\left\{\varphi_{j}\right\}$.

(iv) Set

$$
l_{n i}(x)=\prod_{\substack{j=1 \\ j \neq i}}^{n}\left(\frac{x-x_{n j}}{x_{n i}-x_{n j}}\right), \quad 1 \leqslant i \leqslant n,
$$

so that $L_{n}(g)=\sum_{i=1}^{n} l_{n i}(x) g\left(x_{n i}\right)$ is the Lagrangian polynomial of degree $\leqslant n-1$ interpolating to $g$ at the $\left\{x_{n i}\right\}$. When $g$ has interior singularities, we define

$$
L_{n}^{*}(g)=\sum_{j \in \tau(n, g)} l_{n j}(x) g\left(x_{n j}\right)
$$

with the notation of $(2.3),(2.4 \mathrm{~A}, \mathrm{~B})$. Note that

$$
\lambda_{n j}=\int_{a}^{b} l_{n j}(x) d \alpha(x)=\int_{a}^{b} l_{n j}^{2}(x) d \alpha(x), \quad 1 \leqslant j \leqslant n
$$

(Freud [4, Theorem 1.3.2, p. 21]), and hence,

$$
K_{n}(g)=\int_{a}^{b} L_{n}(g) d \alpha(x), \quad K_{n}^{*}(g)=\int_{a}^{b} L_{n}^{*}(g) d \alpha(x) .
$$

(v) As usual, $\|g\|_{\alpha, p}=\left(\int_{a}^{b}|g(x)|^{p} d \alpha(x)\right)^{1 / p}$ whenever $p \geqslant 1$ and the LebesgueStieltjes integral is defined and finite. For $p=\infty,\|g\|_{\alpha, p}=\sup \{|g(x)|: x \in(a, b)\}$.

(vi) We shall seek to approximate integrals of the form

$$
I[\beta ; f]=\int_{a}^{b} f(x) d \beta(x),
$$

where $\beta:(a, b) \rightarrow \mathbf{R}$ is right continuous and of bounded variation in $(a, b)$ and where, in the Lebesgue-Stieltjes sense,

$$
\int_{a}^{b} t^{j} d \beta(t) \text { exists and is finite, } \quad j=0,1,2, \ldots .
$$


Sloan and Smith [13] considered the case $d \beta(x)=k(x) d x$. The more general form (2.10) allows $d \beta(x)$ to be discrete,so that $I[\beta ; f]$ is a series. Approximating infinite series by rewriting them as Stieltjes integrals, and then using Gaussian quadrature on the Stieltjes integral, is of some practical and theoretical interest.

(vii) We shall require that $\beta(x)$ is absolutely continuous with respect to $\alpha(x)$, that is,

There is a function $d \beta / d \alpha(x)$ (the Radon-Nikodym derivative), defined almost everywhere with respect to $d \alpha(x)$, such that

$$
\beta(x)=\int_{a}^{x} \frac{d \beta}{d \alpha}(u) d \alpha(u), \quad x \in(a, b),
$$

except possibly at discontinuities of $\alpha(x)$.

In the case where $d \beta(x)=k(x) d x$ and $d \alpha(x)=w(x) d x$, we see that $d \beta / d \alpha(x)=$ $k(x) / w(x)$. Thus (2.12) is satisfied if $k(x)$ is integrable and vanishes almost everywhere in $\{x: w(x)=0\}$. One consequence of $(2.12)$ is that whenever $f(x)$ is defined almost everywhere with respect to $d \alpha(x)$ and, whenever $f(x)$ is LebesgueStieltjes integrable in $(a, b)$ with respect to $d \beta(x)$,

$$
\int_{a}^{b} f(x) d \beta(x)=\int_{a}^{b} f(x) \frac{d \beta}{d \alpha}(x) d \alpha(x) .
$$

See Riesz-Sz. Nagy [12, p. 127] for (2.13), though they do not use the name "Radon-Nikodym derivative" - for the latter, see de Barra [2, Chapter 8]. Note also that if $d|\beta|(x)$ is the total variation of $d \beta(x)$, then

$$
\frac{d|\beta|}{d \alpha}(x)=\left|\frac{d \beta}{d \alpha}\right|(x) \text { almost everywhere with respect to } d \alpha(x)
$$

(see de Barra [2, p. 166]) and hence,

$$
\int_{a}^{b} f(x) d|\beta|(x)=\int_{a}^{b} f(x)\left|\frac{d \beta}{d \alpha}\right|(x) d \alpha(x)
$$

whenever $f(x)$ is Lebesgue-Stieltjes integrable in $(a, b)$ with respect to $d \beta(x)$.

(2.12) forces $\beta$ to be continuous at every point of continuity of $\alpha$ (among other things). So, given $\beta(x)$, we have to choose the $\alpha(x)$ on which we base the product integration rule in such a way that $(2.12)$ holds.

(viii) The product rules considered will have the form

$$
I[\beta ; f] \simeq I_{n}[\beta ; f]=\sum_{i=1}^{n} w_{n i} f\left(x_{n i}\right),
$$

where the $\left\{w_{n i}\right\}_{i}$ are chosen to make the rule exact for polynomials of degree less than $n$. It is easy to see that

$$
I_{n}[\beta ; f]=\int_{a}^{b} L_{n}(f) d \beta(x)
$$

and

$$
w_{n i}=\lambda_{n i} S_{n-1}\left[\frac{d \beta}{d \alpha}\right]\left(x_{n i}\right), \quad 1 \leqslant i \leqslant n
$$


Sloan and Smith [13] proved these for $d \beta(x)=k(x) d x$ and $d \alpha(x)=w(x) d x$. Note that in view of $(2.11),(2.13)$,

$$
\int_{a}^{b} t^{j} d \beta(t)=\int_{a}^{b} t^{j} \frac{d \beta}{d \alpha}(t) d \alpha(t)
$$

exists for $j=0,1,2, \ldots$ Hence, the right member of (2.18) is defined. Associated with (2.16), there is the "companion rule", first introduced by Sloan and Smith [13]:

$$
J_{n}[\beta ; f]=\sum_{i=1}^{n}\left|w_{n i}\right| f\left(x_{n i}\right) .
$$

When $f$ has singularities interior to $(a, b)$, we define, with the notation of $(2.4 \mathrm{~A}, \mathrm{~B})$ :

$$
\begin{aligned}
I_{n}^{*}[\beta ; f] & =\sum_{j \in \tau(n, f)} w_{n j} f\left(x_{n j}\right) \\
J_{n}^{*}[\beta ; f] & =\sum_{j \in \tau(n, f)}\left|w_{n j}\right| f\left(x_{n j}\right) .
\end{aligned}
$$

3. Convergence of Gaussian Quadratures. In this section, we use a lemma due to Shohat and a generalized Markov-Stieltjes inequality to prove a convergence theorem for Gaussian quadrature and Lagrange interpolation of singular integrands - Theorem 3.5. This theorem is the corrected version of a result stated by Freud [4, pp. 132-133, Problems 12, 13] which we show, by counterexample, to be incorrect. For functions with endpoint singularities only, detailed results appear in Freud [4, pp. 92-98]. These results are superior to the "dominated integrability" results established in recent years, in the sense that they do not require certain inequalities between all weights and abscissas near the singularity, but the methods work only for Gaussian quadrature.

Lemma 3.1 (Sнонат). Suppose $G(x)$ is infinitely differentiable in $(a, b)$ with

$$
G^{(2 k)}(x) \geqslant 0 \quad \text { in }(a, b), k=0,1,2, \ldots
$$

Then

$$
K_{n}(G) \leqslant \int_{a}^{b} G(x) d \alpha(x), \quad n=0,1,2, \ldots,
$$

provided the integral is convergent.

Proof. See, for example, Freud [4, p. 92]. Q.E.D.

The following lemma is based on a generalized Markov-Stieltjes inequality first proved by Posse (Freud [4, p. 33]).

Lemma 3.2. Suppose $G(x)$ is infinitely differentiable in $(-\infty, \infty) \backslash\{y\}$ with $y \in(a, b)$ and

$$
\begin{aligned}
G^{(k)}(x) \geqslant 0 \quad \text { in }(-\infty, y) ; \quad(-1)^{k} G^{(k)}(x) \geqslant 0 \quad \text { in }(y, \infty), \\
k=0,1,2, \ldots .
\end{aligned}
$$

Then

$$
K_{n}^{*}(G) \leqslant \int_{a}^{b} G(x) d \alpha(x), \quad n=1,2,3, \ldots
$$


More precisely if $y \in\left(x_{n t}, x_{n, t+1}\right)$ some $1 \leqslant t<n$, then

$$
K_{n}(G) \leqslant \int_{a}^{b} G(x) d \alpha(x)+\lambda_{n t} G\left(x_{n t}\right)+\lambda_{n, t+1} G\left(x_{n, t+1}\right),
$$

while if $y=x_{n, t+1}$, some $0 \leqslant t<n$, and we set $G(y)=0$, then

$$
K_{n}(G) \leqslant \int_{a}^{b} G(x) d \alpha(x) .
$$

Proof. Suppose first $y \in\left(x_{n t}, x_{n, t+1}\right)$ for some $0 \leqslant t \leqslant n$ (where $a=x_{n 0}, b=$ $\left.x_{n, n+1}\right)$.

Now, apply the following inequality due to Posse (Freud [4, p. 33]) with $\xi=x_{n t}$ :

$$
\sum_{x_{n j}<\xi} \lambda_{n j} G\left(x_{n j}\right) \leqslant \int_{-\infty}^{\xi} G(x) d \alpha(x) \leqslant \sum_{x_{n j} \leqslant \xi} \lambda_{n j} G\left(x_{n j}\right),
$$

so that

$$
\sum_{j=1}^{t-1} \lambda_{n j} G\left(x_{n j}\right) \leqslant \int_{-\infty}^{x_{n t}} G(x) d \alpha(x)
$$

(the sum is taken as 0 if $t=0,1$ ). Note that Freud proves (3.4) under the stronger assumption that strict inequality holds in (3.1), but as he remarks [4, p. 50, Problem 16] consideration of $G_{\varepsilon}(x)=G(x)+\varepsilon e^{x}$ with $\varepsilon \rightarrow 0+$ gives (3.4) under the weaker assumption $G^{(k)}(x) \geqslant 0$ in $(-\infty, y), k=0,1,2, \ldots$.

To deal with $\sum_{j=t+2}^{n} \lambda_{n j} G\left(x_{n j}\right)$, we consider the transformation $x \rightarrow-x$. It is easy to see, that for the distribution function $\gamma(x)=\alpha(\infty)-\alpha(-x)$, the orthonormal polynomials are the $\left\{\varphi_{j}(-x)\right\}$. Further, the abscissas $z_{n j}$ and weights $\mu_{n j}$ in the quadrature formula with respect to $\gamma$ satisfy $z_{n j}=-x_{n, n+1-j}$ and $\mu_{n j}=\lambda_{n, n+1-j}$, $j=1,2, \ldots, n$. Next, $H(x)=G(-x)$ satisfies

$$
H^{(i)}(x)=(-1)^{i} G(-x) \geqslant 0 \text { for } x \in(-\infty,-y), i=0,1,2, \ldots .
$$

Setting $s=n-t$ and $\xi=z_{n s}=-x_{n, t+1}$ in Posse's inequality yields

$$
\begin{gathered}
\sum_{z_{n j}<\xi} \mu_{n j} H\left(z_{n j}\right) \leqslant \int_{-\infty}^{\xi} H(x) d \gamma(x) \leqslant \sum_{z_{n j} \leqslant \xi} \mu_{n j} H\left(z_{n j}\right), \\
\sum_{j=t+2}^{n} \lambda_{n j} G\left(x_{n j}\right) \leqslant \int_{x_{n, t+1}}^{\infty} G(x) d \alpha(x)
\end{gathered}
$$

(the sum is taken as 0 if $t=n-1, n$ ). Next, if $t>0$ and

$$
\alpha(y)-\alpha\left(x_{n t}\right) \geqslant \lambda_{n t},
$$

then monotonicity of $G$ in $(-\infty, y)$ gives

$$
\lambda_{n t} G\left(x_{n t}\right) \leqslant \int_{x_{n t}}^{y} G(x) d \alpha(x),
$$

while if $t<n$ and

$$
\alpha\left(x_{n, t+1}\right)-\alpha(y) \geqslant \lambda_{n, t+1},
$$

then monotonicity of $G$ in $(y, \infty)$ gives

$$
\lambda_{n, t+1} G\left(x_{n, t+1}\right) \leqslant \int_{y}^{x_{n, t+1}} G(x) d \alpha(x) .
$$


If both (3.6), (3.8) hold, then (3.4), (3.5), (3.7), (3.9) give (3.2), (3.3), as $G$ is nonnegative. If (3.6) (respectively (3.8)) fails, then (2.4B) shows that $x_{n t}$ (respectively $\left.x_{n, t+1}\right)$ is omitted in forming the sum $K_{n}^{*}(G)$ and (3.2), (3.3) still follow from (3.4), (3.5).

Finally, if $y=x_{n, t+1}$, we apply the inequality of Posse with $\xi=x_{n, t+1}$ to obtain

$$
\sum_{j=1}^{t} \lambda_{n j} G\left(x_{n j}\right) \leqslant \int_{-\infty}^{x_{n, t+1}} G(x) d \alpha(x)
$$

and the inequality

$$
\sum_{j=t+2}^{n} \lambda_{n j} G\left(x_{n j}\right) \leqslant \int_{x_{n, t+1}}^{\infty} G(x) d \alpha(x)
$$

follows from (3.10), as before, by considering the transformation $x \rightarrow-x$. Q.E.D.

The function $G(x)$ in Lemma 3.2 is "absolutely monotone" in $(-\infty, y)$ and "completely monotone" in $(y, \infty)$. This, together with the above lemmas, motivates the following definition.

Definition 3.3. We say that $g(x)$ is monotone integrable on $(a, b)$ if

(a) there exists a nonnegative integer $l$ and points $a=y_{0}<y_{1}<\cdots<y_{l}<y_{l+1}$ $=b$ such that $\alpha$ is continuous and strictly increasing from the left and right at $y_{1}, \ldots, y_{l}$, and $g$ is properly Riemann-Stieltjes integrable with respect to $d \alpha(x)$ in each compact subinterval of $\left(y_{i}, y_{i+1}\right), i=0,1, \ldots, l$ with

$$
\lim _{\substack{A \rightarrow y_{i}+\\ B \rightarrow y_{i+1}}} \int_{A}^{B}|g(x)| d \alpha(x)<\infty, \quad i=0,1, \ldots, l
$$

(where $(-\infty)+\equiv-\infty ; \infty-\equiv \infty)$.

(b) there exist functions $G_{i}(x), i=0,1, \ldots, l+1$ such that

$$
\underset{\substack{x \rightarrow y_{i} \\ x \in(a, b)}}{\limsup }\left|g(x) / G_{i}(x)\right|<\infty, \quad i=0,1, \ldots, l+1,
$$

where, for $i=0$ and $i=l+1$,

$$
\left\{\begin{array}{l}
G_{i}(x) \text { is infinitely differentiable in }(a, b) \\
\text { with } G_{i}^{(2 k)}(x) \geqslant 0 \text { in }(a, b), k=0,1,2, \ldots,
\end{array}\right.
$$

but for $i=1,2, \ldots, l$,

$$
\left\{\begin{array}{l}
G_{i}(x) \text { is infinitely differentiable in }(-\infty, \infty) \backslash\left\{y_{i}\right\}, \text { with } \\
G_{i}^{(k)}(x) \geqslant 0 \text { in }\left(-\infty, y_{i}\right) ;(-1)^{k} G_{i}^{(k)}(x) \geqslant 0 \text { in }\left(y_{i}, \infty\right), k=0,1,2, \ldots,
\end{array}\right.
$$

and where

$$
\int_{a}^{b} G_{i}(x) d \alpha(x)<\infty, \quad i=0,1, \ldots, l+1 .
$$

Instead of (3.12), Freud assumes more-namely, that the lim sup in (3.12) is 0 . Esser [3] was the first to notice (for endpoint singularities) that it suffices to have the lim sup finite. By saying $\alpha$ is strictly increasing from the left and right at $y_{1}, \ldots, y_{l}$, we mean that for $i=1,2, \ldots, l$, there is an open interval containing $y_{i}$ in which $\alpha$ is strictly increasing. This is a natural requirement - if, for example, $\alpha$ were constant near $y_{i}$, we could alter the values of $g$ near $y_{i}$ without affecting $\int_{a}^{b} g(x) d \alpha(x)$. 
A natural choice for $G_{i}(x), i=1,2, \ldots, l$, is $G_{i}(x)=\left|x-y_{i}\right|^{-\delta}$, for some $0<\delta$ $<1$. In fact, one of the authors [6, Corollary 4] has shown that given $\varepsilon>0$, and provided $\alpha^{\prime}(x)$ is bounded in a neighborhood of $y_{i}, i=1,2, \ldots, l$, one may choose $G_{i}(x)$ satisfying (b) and growing like $\left|x-y_{i}\right|^{-1}\left(\log \left|x-y_{i}\right|\right)^{-1-\varepsilon}$ as $x \rightarrow y_{i}$. Further, for weights such as $d \alpha(x)=\exp \left(-|x|^{\lambda}\right) d x, x \in \mathbf{R}, \lambda \geqslant 1$, Corollary 2 in [6] shows that one may choose $G_{0}(x)$ and $G_{l+1}(x)$ satisfying (b) and growing like $\exp \left(|x|^{\lambda}\right)|x|^{-1-\varepsilon}$ as $|x| \rightarrow \infty$, for some $\varepsilon>0$. The following lemma lists some closure properties of the class of monotone integrable functions.

LEMMA 3.4. (a) Let $f(x)$ be properly Riemann-Stieltjes integrable with respect to $d \alpha(x)$ in each compact subinterval of $(a, b)$. Further, suppose there exist $C, D>0$ and a positive even integer s such that $|f(x)| \leqslant C+D x^{s}$ for all $x \in(a, b)$. Then $f(x)$ is monotone integrable.

(b) If $f(x), g(x)$ are monotone integrable and $c, d \in \mathbf{R}$, then so are $c f(x)+d g(x)$, and $|f(x)|$.

(c) If $f^{2}(x)$ is monotone integrable, and $P(x)$ is a polynomial, then $(f|P|)(x)$ and $(f-P)^{2}(x)$ are monotone integrable.

Proof. (a) Since $f(x)$ has no singularities in $(a, b)$, we have $l=0$ in Definition 3.3 and can clearly choose $G_{0}(x)=G_{l+1}(x)=C+D x^{s}$.

(b) Is easy.

(c) As $g(x)=f^{2}(x)$ is monotone integrable, there exist $\left\{y_{i}\right\},\left\{G_{i}\right\}$ as in Definition 3.3. Using Hölder's inequality, and (2.1), we see $f|P|$ satisfies (3.11). Further, the $G_{1}, G_{2}, \ldots, G_{l}$ that satisfy (3.12), (3.14), (3.15) for $g=f^{2}$ also satisfy (3.12), (3.14), (3.15) for $g=f|P|$, as

$$
\limsup _{x \rightarrow y_{i}}\left|(f|P|)(x) / G_{i}(x)\right|=\limsup _{x \rightarrow y_{i}}\left|\left[f^{2}(x) / G_{i}(x)\right][|P|(x) / f(x)]\right|<\infty .
$$

We need modify only $G_{0}(x)$ (respectively $G_{l+1}(x)$ ) and only in the case that $a$ (respectively $b$ ) are infinite. Suppose, for example, $a=-\infty$. Choose an even integer $s$ larger than the degree of $P^{2}$, and consider $G(x)=G_{0}(x)+x^{s}$, which obviously satisfies (3.13) and (3.15). Now

$$
\begin{aligned}
|G(x) /(f|P|)(x)| & =\left[G_{0} / f^{2}\right](x)|f / P|(x)+\left[x^{s} / P^{2}(x)\right]|P / f|(x) \\
& \geqslant \min \left\{G_{0}(x) / f^{2}(x), x^{s} / P^{2}(x)\right\} \\
& \geqslant c>0 \text { for large negative } x .
\end{aligned}
$$

Hence, we can use $G(x)$ as $G_{0}(x)$ for $g=f|P|$. Finally, $(f-P)^{2}=f^{2}-2 f P+P^{2}$ is a linear combination of monotone integrable functions. Q.E.D.

THEOREM 3.5. (a) Let $g(x)$ be monotone integrable. Then

$$
\lim _{n \rightarrow \infty} K_{n}^{*}(g)=\int_{a}^{b} g(x) d \alpha(x) .
$$

(b) Let $g^{2}(x)$ be monotone integrable. Then,

$$
\lim _{n \rightarrow \infty}\left\|L_{n}^{*}(g)-g\right\|_{\alpha, 2}=0 .
$$


Proof. For functions with no interior singularities, these results are Theorem III.1.6(a), (b) and Theorem III.2.2 in Freud [4, pp. 94, 97].

(a) We assume the $l,\left\{y_{i}\right\},\left\{G_{i}\right\}$ are as in Definition 3.3. Fix $y=y_{i}$, some $1 \leqslant i \leqslant l$. For each $n \geqslant 1$, let $j_{n}$ denote the positive integer such that $y \in$ $\left(x_{n, j_{n}-1}, x_{n, j_{n}+1}\right)$. We claim that for any positive integer $r$,

$$
\lim _{n \rightarrow \infty} x_{n, j_{n}-r}=y=\lim _{n \rightarrow \infty} x_{n, j_{n}+r}=y
$$

(where $x_{n k}=a$ for $k \leqslant 0, x_{n k}=b$ for $k>n$ ). If, say, the first limit fails to hold for some $r$, we can find a subsequence of integers $\mathscr{S}$ and $\delta>0$ such that $\lim _{n \rightarrow \infty: n \in \mathscr{S}} x_{n, j_{n}-k}=y, k=1,2, \ldots, r-1$, but $x_{n, j_{n}-r}<y-\delta$ all $n \in \mathscr{S}$. Then, for any $0<\eta<\delta$, we have

$$
\lim _{\substack{n \rightarrow \infty \\ n \in \mathscr{S}}} \sum_{x_{n p} \in[y-\delta, y-\eta]} \lambda_{n p}=0
$$

But assuming (as we can) that $y-\delta, y-\eta$ are points of continuity of $\alpha$, the limit in (3.17) should be $\alpha(y-\eta)-\alpha(y-\delta)$ (by Theorem III.1.1 in Freud [4, p. 89]). As $\alpha$ is continuous and strictly increasing from the left at $y$, we have $\alpha(y-\eta)-$ $\alpha(y-\delta)>0$ for small $\eta$, and so we have a contradiction to (3.17). Hence (3.16) holds. Next, by right continuity of $\alpha$ and the Markov-Stieltjes inequality (see, for example, Freud [4, p. 29]), we have

$$
\lambda_{n j_{n}} \leqslant \alpha\left(x_{n, j_{n}+1}\right)-\alpha\left(x_{n, j_{n}-1}\right) \rightarrow 0 \text { as } n \rightarrow \infty,
$$

by (3.16) and continuity of $\alpha$ at $y$. Since $\tau(n, g)$ omits at most $2 l$ integers, and $j \notin \tau(n, g)$ implies $y_{i} \in\left(x_{n, j-1}, x_{n, j+1}\right)$ for some $1 \leqslant i \leqslant l$, we have

$$
\lim _{n \rightarrow \infty} \sum_{j \notin \tau(n, g)} \lambda_{n j}=0 \text {. }
$$

Theorem III.1.1 in Freud [4, p. 89] and (3.18) imply

$$
\lim _{n \rightarrow \infty} \sum_{j \in \tau(n, g)} \lambda_{n j} h\left(x_{n j}\right)=\int_{a}^{b} h(x) d \alpha(x)
$$

for any function $h$ bounded in $(a, b)$ and properly Riemann-Stieltjes integrable with respect to $d \alpha(x)$ in each compact subinterval of $(a, b)$ and such that $h$ is (improperly) Riemann-Stieltjes integrable with respect to $d \alpha(x)$ in $(a, b)$.

Next, by (3.12), we can choose $K>0$ and open intervals, $\mathscr{I}_{1}, \mathscr{I}_{2}, \ldots, \mathscr{I}_{l}$ containing $y_{1} \cdots y_{l}$ and (possibly unbounded) open intervals $\mathscr{I}_{0}$ with left endpoint $a$, $\mathscr{I}_{1+1}$ with right endpoint $b$, such that

$$
|g(x)| \leqslant K G_{i}(x), \quad x \in \mathscr{I}_{i}, i=0,1, \ldots, l+1 .
$$

By reducing the sizes of the $\mathscr{I}_{i}$, if necessary, we may assume that for $\mathscr{I}=\bigcup_{i=0}^{l+1} \mathscr{I}_{i}$, and for some given $\varepsilon>0$,

$$
K \sum_{i=0}^{1+1} \int_{\mathscr{C}_{1}} G_{i}(x) d \alpha(x)<\varepsilon .
$$

In view of (3.20), this implies that

$$
\left|\int_{\mathscr{g}} g(x) d \alpha(x)\right|<\varepsilon .
$$


Let

$$
\chi(x)= \begin{cases}1, & x \in(a, b) \backslash \mathscr{I} \\ 0, & \text { otherwise }\end{cases}
$$

Then (2.5) gives

$$
\begin{aligned}
\left|K_{n}^{*}(g)-\int_{a}^{b} g(x) d \alpha(x)\right| & \\
\leqslant & \left|\sum_{j \in \tau(n, g)} \lambda_{n j}(g \chi)\left(x_{n j}\right)-\int_{a}^{b}(g \chi)(x) d \alpha(x)\right| \\
& +\left|\sum_{\substack{j \in \tau(n, g) \\
x_{n j} \in \mathscr{I}}} \lambda_{n j} g\left(x_{n j}\right)\right|+\left|\int_{\mathscr{I}} g(x) d \alpha(x)\right| .
\end{aligned}
$$

Since $h(x)=(g \chi)(x)$ is bounded in $(a, b) \backslash \mathscr{I}$ and vanishes outside this bounded set, (3.19) shows that the first term in the right member of (3.22) tends to 0 as $n \rightarrow \infty$. Next, by (3.21B), the third term in the right member of (3.22) is bounded by $\varepsilon$. Finally, we deal with the second term. By (3.20) and the definition of $\chi$,

$$
\begin{aligned}
& \left|\sum_{\substack{j \in \tau(n, g) \\
x_{n j} \in \mathcal{I}}} \lambda_{n j} g\left(x_{n j}\right)\right| \leqslant K \sum_{i=0}^{l+1} \sum_{j \in \tau(n, g)} \lambda_{n j}\left(G_{i}(1-\chi)\right)\left(x_{n j}\right) \\
& \leqslant K \sum_{i=0}^{l+1}\left\{\sum_{j \in \tau\left(n, G_{i}\right)} \lambda_{n j} G_{i}\left(x_{n j}\right)-\sum_{j \in \tau(n, g)} \lambda_{n j}\left(G_{i} \chi\right)\left(x_{n j}\right)\right\} \\
& \quad\left(\text { as } \tau(n, g) \subset \tau\left(n, G_{i}\right) \text { and } G_{i} \text { is nonnegative }\right) \\
& \leqslant K \sum_{i=0}^{l+1}\left\{\int_{a}^{b} G_{i}(x) d \alpha(x)-\int_{a}^{b}\left(G_{i} \chi\right)(x) d \alpha(x)+\varepsilon /(K(l+2))\right\}
\end{aligned}
$$

(for large $n$, by Lemma 3.2, (2.5) and (3.19))

$\leqslant 2 \varepsilon$ for large $n$, by $(3.21 \mathrm{~A})$.

This completes the proof of (a).

(b) Let $\varepsilon>0$. As $d \alpha(x)$ is the unique solution of its Hamburger moment problem, the same is true of $d \alpha(x) /\left(1+x^{2}\right)$ [4, p. 77, Proof of Theorem II.4.3]. Then, by a theorem of M. Riesz [4, p. 77] there exists a polynomial $P(x)$ such that $\|g-P\|_{\alpha, 2}$ $<\varepsilon$. Now, if $n$ exceeds degree $(P)$, we have $P=L_{n}(P)$ and so (2.7) and Minkowski's inequality give

$$
\begin{aligned}
\left\|L_{n}^{*}(g)-g\right\|_{\alpha, 2} \leqslant & \sum_{j \in \tau(n, g)} l_{n j}(x)(g-P)\left(x_{n j}\right) \|_{\alpha, 2} \\
& +\left\|\sum_{j \notin \tau(n, g)} l_{n j}(x) P\left(x_{n j}\right)\right\|_{\alpha, 2}+\|P-g\|_{\alpha, 2} .
\end{aligned}
$$

Writing

$$
h_{n}(x)= \begin{cases}0 & \text { if } x=x_{n j} \text { some } j \notin \tau(n, g), \\ (g-P)(x) & \text { all other } x\end{cases}
$$


we see that the first term in the right member of (3.23) is $\left\|L_{n}\left(h_{n}\right)\right\|_{\alpha, 2}$. Now, by exactness of the rule (2.2), we have

$$
\left\|L_{n}\left(h_{n}\right)\right\|_{\alpha, 2}^{2}=\int_{a}^{b} L_{n}^{2}\left(h_{n}\right)(x) d \alpha(x)=K_{n}\left(h_{n}^{2}\right)=K_{n}^{*}\left((g-P)^{2}\right)
$$

(by (3.24) and as $\left.\tau\left(n,(g-P)^{2}\right)=\tau(n, g)\right)$

$$
\rightarrow \int_{a}^{b}(g-P)^{2}(x) d \alpha(x)=\|g-P\|_{\alpha, 2}^{2} \quad \text { as } n \rightarrow \infty .
$$

Here we have used Theorem 3.5(a) and Lemma 3.4(c). Next, applying Hölder's inequality to the second term in the right member of (3.23) and using $\lambda_{n j}=\left\|l_{n j}\right\|_{\alpha, 2}^{2}$ $((2.8))$, we see that

$$
\left\|\sum_{j \notin \tau(n, g)} l_{n j}(x) P\left(x_{n j}\right)\right\|_{\alpha, 2}^{2} \leqslant\left\{\sum_{j \notin \tau(n, g)} \lambda_{n j}\right\}\left\{\sum_{j \notin \tau(n, g)} P^{2}\left(x_{n j}\right)\right\} \rightarrow 0 \text { as } n \rightarrow \infty
$$

(by (3.18) and boundedness of $P$ in $\left[y_{1}-\delta, y_{l}+\delta\right]$ any $\delta>0$, and as $\tau(n, g)$ omits at most $2 l$ integers). Together with (3.23), (3.25), this completes the proof of the theorem. Q.E.D.

Remarks. (a) Theorem 3.5(a) deals with very general weight functions and integrands, and as such, is not included in the work of Rabinowitz [10] or of Lubinsky and Rabinowitz [7]. In the latter paper, the authors showed that if $\alpha^{\prime}(x)$ is bounded above and below by positive constants near $y \in(a, b)$, then

$$
\int_{a}^{b}|x-y|^{-\delta} d \alpha(x)-K_{n}^{*}\left(|x-y|^{-\delta}\right)
$$

tends to zero like $n^{-1+\delta}$ as $n \rightarrow \infty$ when $(a, b)$ is bounded-see Theorem 4.3, Corollary 4.4 and Theorem 7.5 in [7]. Although $(a, b)$ was bounded in [7], the methods there extend to infinite intervals.

Further, because of the stronger assumptions on $d \alpha(x)$ in [7] (though these were "local" assumptions), one could replace $(2.4 \mathrm{~A}, \mathrm{~B})$ by the simpler condition that the closest abscissa to each interior singularity is omitted. Consequently, for the weight functions in [7], Theorem 3.5 holds with the simpler definition of $K_{n}^{*}(g)$ and $L_{n}^{*}(g)$ that only the closest term to each singularity is omitted. This is the case not only for finite intervals, but for weight functions on the whole real line such as $\exp \left(-|x|^{\lambda}\right)$ $(\lambda \geqslant 1)$ or even Freud's weight functions [5].

(b) We show now (compare Davis and Rabinowitz [1]) that the assertion of Problem 12 in Freud [4, pp. 132-133] is incorrect. Freud evidently intended to ignore the singularity by setting $f\left(x_{n j}\right)=0$ whenever $x_{n j}$ coincided with a singularity of $f$. For simplicity, let $(a, b)$ be finite and $\alpha^{\prime}(x)$ be bounded above and below by positive constants in an open interval containing some closed subinterval $J$ of $(a, b)$. By Corollary 4.4(iii) in [7], for $y \in J$,

$$
\begin{aligned}
& \int_{a}^{b}|x-y|^{-\delta} d \alpha(x)-K_{n}\left[|x-y|^{-\delta}\right] \\
& \leqslant-\varepsilon n^{-1}\left|x_{c(n)}-y\right|^{-\delta}+O\left(n^{-1+\delta}\right),
\end{aligned}
$$

where $x_{c(n)}$ is the closest abscissa to $y$ and $\varepsilon$ is independent of $n$ and $y$. Since the abscissas $\left\{x_{n j}\right\}$ are dense in $J$, it is easy to see that we can choose $y \in J$ such that $0<\left|x_{c(n)}-y\right|<e^{-n}$ for infinitely many $n$. Consequently the left member in (3.26) 
diverges to $-\infty$ for an infinite subsequence of integers. By a lengthier argument, it is possible to give a similar counterexample for more general weights $d \alpha(x)$, for which $\alpha(x)$ is strictly increasing in some subinterval of the support of $d \alpha$, regardless of whether the support is bounded or unbounded.

(c) For weights on the whole real line, there is the following corollary:

COROllary 3.6. Let $P(x)$ be a polynomial, not vanishing identically. Let $\lambda \geqslant 1$ and $\gamma>-1$. Let

$$
\alpha^{\prime}(x)=|P(|x|)|^{\gamma} \exp \left(-|x|^{\lambda}\right), \quad x \in \mathbf{R} .
$$

Let $f: \mathbf{R} \rightarrow \mathbf{R}$ and $-\infty=y_{0}<y_{1}<y_{2}<\cdots<y_{1+1}=\infty$ be such that $f$ is bounded and Riemann integrable in each compact subinterval of $\left(y_{i}, y_{i+1}\right), i=0,1,2, \ldots, l$. Further assume $P\left(\left|y_{i}\right|\right) \neq 0, i=1,2, \ldots, l$.

(i) Suppose $|f(x)| \alpha^{\prime}(x)$ and $f(x) \alpha^{\prime}(x)$ are improperly Riemann integrable in $\mathbf{R}$ and that

$$
\begin{gathered}
\lim _{x \rightarrow y_{i}}|f(x)|\left|x-y_{i}\right||\log | x-y_{i}||^{2}=0, \quad i=1,2, \ldots, l . \\
\lim _{|x| \rightarrow \infty}|f(x)||P(|x|)|^{\gamma} \exp \left(-|x|^{\lambda}\right)\left|x\|\log \mid x\|^{2}=0 .\right.
\end{gathered}
$$

Then,

$$
\lim _{n \rightarrow \infty} K_{n}^{*}[f]=\int_{-\infty}^{\infty} f(x) \alpha^{\prime}(x) d x .
$$

(ii) Assume $f^{2}(x) \alpha^{\prime}(x)$ is improperly Riemann integrable in $\mathbf{R}$ and that (3.28) and (3.29) hold with $|f|$ replaced by $f^{2}$. Then,

$$
\lim _{n \rightarrow \infty}\left\|L_{n}^{*}(f)-f\right\|_{\alpha, 2}=0
$$

Proof. (i) We note firstly that $d \alpha(x)$ is the unique solution of its Hamburger moment problem (see Theorem II.5.2 in [4, p. 80]). Further, we can write

$$
\alpha^{\prime}(x)=\exp (-2 Q(|x|)), \quad x \in \mathbf{R},
$$

where

$$
Q(x)=\left\{|x|^{\lambda}-\gamma \log |P(|x|)|\right\} / 2, \quad x \in \mathbf{R} .
$$

As $\lambda \geqslant 1$, we see that $Q^{\prime}(x)>0$ for large $x$, and that (7) in the definition in [6] is satisfied with arbitrarily small $\theta>0$. Hence, in the sense of [6], $d \alpha$ is a Freud weight. It follows from Corollary 2 in [6], that there exists $G_{0}=G_{l+1}$ satisfying (3.13) and (3.15) and such that for some positive $c$, and for large $|x|$,

$$
G_{0}(x) \geqslant\left. c|P(|x|)|^{-\gamma} \exp \left(|x|^{\lambda}\right)|x|^{-1}|\log | x\right|^{-2} \text {. }
$$

Then (3.29) implies that (3.12) holds for $i=0$ and $i=l+1$. Next, Corollary 4 in [6] shows that there exist $G_{1}, G_{2}, \ldots, G_{l}$ satisfying (3.14), (3.15) with

$$
G_{i}(x) \geqslant c\left|x-y_{i}\right|^{-1}|\log | x-y_{i}||^{-2}, \quad i=1,2, \ldots, l .
$$

Then (3.29) implies (3.12) for $i=1,2, \ldots$, l. Hence, Theorem 3.5(a) yields our result.

(ii) is similar. Q.E.D. 
We note that $\|\left.\log \left|x-y_{i}\right|\right|^{2}$ in (3.28) can be replaced by $\|\left.\log \left|x-y_{i}\right|\right|^{1+\varepsilon}$ or $\| \log \left|x-y_{i}\right||| \log |\log | x-\left.y_{i}||\right|^{1+\varepsilon}$ and so on, for any $\varepsilon>0$; see [6]. Similarly, $|\log | x||^{2}$ in (3.29) can be replaced by $|\log | x||^{(1+\varepsilon)}$ or $\left\|\log \left|x\||\log | \log |x|\|^{(1+\varepsilon)}\right.\right.$ and so on, for any $\varepsilon>0$.

4. Convergence of Product Integration Rules. Theorem 3.5 enables us to prove convergence of product integration rules based on Gaussian quadratures. First, however, we need the following lemma:

Lemma 4.1. (a) Assume $f$ is monotone integrable. Then, if $1 \leqslant p, q \leqslant \infty$ and $1 / p+1 / q=1$, we have

$$
\left|I_{n}^{*}[\beta ; f]-\int_{a}^{b} f(x) d \beta(x)\right| \leqslant\left\|L_{n}^{*}(f)-f\right\|_{\alpha, p}\left\|\frac{d \beta}{d \alpha}\right\|_{\alpha, q}
$$

provided the right member is finite.

(b) If $f^{2}$ is monotone integrable, and $P$ is any polynomial of degree $\leqslant n$, then

$$
\begin{aligned}
\left|J_{n}^{*}[\beta ; f]-\int_{a}^{b} f(x) d\right| \beta|(x)| \\
\leqslant\left\|P-\frac{d \beta}{d \alpha}\right\|_{\alpha, 2}\left\{\left[K_{n}^{*}\left(f^{2}\right)\right]^{1 / 2}+\|f\|_{\alpha, 2}\right\} \\
\quad+\left|K_{n}^{*}(f|P|)-\int_{a}^{b} f(x)\right| P(x)|d \alpha(x)|
\end{aligned}
$$

provided the right member is finite.

Proof. (a) By definition of $I_{n}^{*}, L_{n}^{*}$ at (2.20), (2.7) we see that

$$
\begin{aligned}
I_{n}^{*}[\beta ; f]-\int_{a}^{b} f(x) d \beta(x) & =\int_{a}^{b}\left(L_{n}^{*}(f)-f\right)(x) d \beta(x) \\
& =\int_{a}^{b}\left(L_{n}^{*}(f)-f\right)(x) \frac{d \beta}{d \alpha}(x) d \alpha(x) .
\end{aligned}
$$

Note that as $f$ is monotone integrable, its points of discontinuity have $d \alpha$-measure zero, and hence $d \beta$-measure zero, by absolute continuity of $\beta$ with respect to $\alpha$. Hölder's inequality then gives (4.1) and that $f$ is Lebesgue-Stieltjes integrable with respect to $d \beta(x)$, provided the norms in (4.1) are finite.

(b) The method of proof is very similar to that of Theorem 1 in Sloan and Smith [13]. Using (2.6), (2.15), (2.18) (2.21) and the fact that $P\left(x_{n j}\right)=S_{n}[P]\left(x_{n j}\right)=$ $S_{n-1}[P]\left(x_{n j}\right)$ (as degree $(P) \leqslant n$ and $\left.\varphi_{n}\left(x_{n j}\right)=0\right)$, we see

$$
\begin{aligned}
&\left|J_{n}^{*}[\beta ; f]-\int_{a}^{b} f(x) d\right| \beta|(x)| \\
& \leqslant\left|\sum_{j \in \tau(n, f)} \lambda_{n j}\left(\left|S_{n-1}\left[\frac{d \beta}{d \alpha}\right]\right|-\left|S_{n-1}[P]\right|\right\}\left(x_{n j}\right) f\left(x_{n j}\right)\right| \\
&+\left|\sum_{j \in \tau(n, f)} \lambda_{n j}(|P| f)\left(x_{n j}\right)-\int_{a}^{b}(|P| f)(x) d \alpha(x)\right| \\
&+\left|\int_{a}^{b} f(x)\left(|P(x)|-\left|\frac{d \beta}{d \alpha}(x)\right|\right) d \alpha(x)\right| .
\end{aligned}
$$


Now

$$
\begin{aligned}
& || S_{n-1}\left[\frac{d \beta}{d \alpha}\right]\left|\left(x_{n j}\right)-\right| S_{n-1}[P]\left|\left(x_{n j}\right)\right| \\
& \quad \leqslant\left|S_{n-1}\left[\frac{d \beta}{d \alpha}\right]-S_{n-1}[P]\right|\left(x_{n j}\right)=\left|S_{n-1}\left[\frac{d \beta}{d \alpha}-P\right]\right|\left(x_{n j}\right) .
\end{aligned}
$$

Then Hölder's inequality gives

$$
\begin{aligned}
\left|\sum_{j \in \tau(n, f)} \lambda_{n j}\left\{\left|S_{n-1}\left[\frac{d \beta}{d \alpha}\right]\right|-\left|S_{n-1}[P]\right|\right\}\left(x_{n j}\right) f\left(x_{n j}\right)\right|^{2} \\
\leqslant\left\{\sum_{j=1}^{n} \lambda_{n j} S_{n-1}^{2}\left[\frac{d \beta}{d \alpha}-P\right]\left(x_{n j}\right)\right\}\left\{\sum_{j \in \tau(n, f)} \lambda_{n j} f^{2}\left(x_{n j}\right)\right\} \\
=\left\{\int_{a}^{b} S_{n-1}^{2}\left[\frac{d \beta}{d \alpha}-P\right](x) d \alpha(x)\right\}\left\{K_{n}^{*}\left(f^{2}\right)\right\} \\
\leqslant\left\|\frac{d \beta}{d \alpha}-P\right\|_{\alpha, 2}^{2}\left\{K_{n}^{*}\left(f^{2}\right)\right\}
\end{aligned}
$$

(by Bessel's inequality). Next as $\tau(n, f|P|)=\tau(n, f)$, the sum in the second term in the right member of (4.3) equals $K_{n}^{*}(f|P|)$. Finally, the third term in the right member of $(4.3)$ is bounded by

$$
\int_{a}^{b}|f(x)|\left|P-\frac{d \beta}{d \alpha}\right|(x) d \alpha(x) \leqslant\|f\|_{\alpha, 2}\left\|P-\frac{d \beta}{d \alpha}\right\|_{\alpha, 2} \text {. Q.E.D. }
$$

We can now prove our main result. For the reader's convenience, the main assumptions on $\alpha, \beta$ are restated.

THEOREM 4.2. Assume that $\alpha:(a, b) \rightarrow \mathbf{R}$ is right continuous, monotone increasing with infinitely many points of increase, and is the unique solution, apart from normalization, of the moment problem (2.1). Assume that $\beta:(a, b) \rightarrow \mathbf{R}$ is right continuous, of bounded variation in $(a, b)$, and is absolutely continuous with respect to $\alpha$, as at (2.12). Suppose that the Radon-Nikodym derivative $d \beta / d \alpha$ satisfies

$$
\left\|\frac{d \beta}{d \alpha}\right\|_{\alpha, 2}<\infty \text {. }
$$

Then, whenever $f^{2}$ is monotone integrable,

$$
\lim _{n \rightarrow \infty} I_{n}^{*}[\beta ; f]=\int_{a}^{b} f(x) d \beta(x)
$$

and

$$
\lim _{n \rightarrow \infty} J_{n}^{*}[\beta ; f]=\int_{a}^{b} f(x) d|\beta|(x)
$$

Proof. Firstly, note that (2.1), (4.6) and Hölder's inequality imply that (2.11) holds. Further, as in the proof of Lemma $4.1, f^{2}$ is Lebesgue-Stieltjes integrable with respect to $d \beta(x)$. Then, Theorem 3.5(b), Lemma 4.1(a) with $p=q=2$ and (4.6) give (4.7). Next, let $\varepsilon>0$. Since $d \beta / d \alpha$ satisfies (4.6), then as in the remarks at the beginning of the proof of Theorem 3.5(b), we can choose a polynomial $P(x)$ such that $\|P-d \beta / d \alpha\|_{\alpha, 2}<\varepsilon$, while as $f^{2}$ is monotone integrable, Theorem 3.5(a) 
gives

$$
\lim _{n \rightarrow \infty} K_{n}^{*}\left(f^{2}\right)=\int_{a}^{b} f^{2}(x) d \alpha(x)=\|f\|_{\alpha, 2}^{2}
$$

Hence the first term in the right member of (4.2) can be bounded by $3 \varepsilon\|f\|_{\alpha, 2}$ for large $n$. Finally, by Lemma $3.4(\mathrm{c}),(f|P|)(x)$ is monotone integrable, so by Theorem 3.5(a),

$$
\lim _{n \rightarrow \infty} K_{n}^{*}(f|P|)=\int_{a}^{b}(f|P|)(x) d \alpha(x)
$$

and this deals with the second term in the right member of (4.2). Q.E.D.

For functions with at worst endpoint singularities, there is the following corollary:

Theorem 4.3. Assume that $\alpha, \beta$ are as in Theorem 4.2, and (4.6) holds. Let $f$ be properly Riemann-Stieltjes integrable, with respect to $d \alpha(x)$, in every compact subinterval of $(a, b)$ and let

$$
\lim _{\substack{A \rightarrow a+\\ B \rightarrow b-}} \int_{A}^{B} f^{2}(x) d \alpha(x)=\|f\|_{\alpha, 2}^{2}<\infty .
$$

Suppose there exist functions $G_{0}, G_{1}$ satisfying (3.13), (3.15) and

$$
\limsup _{x \rightarrow a+} f^{2}(x) / G_{0}(x)<\infty ; \quad \limsup _{x \rightarrow b-} f^{2}(x) / G_{1}(x)<\infty .
$$

Then

$$
\begin{aligned}
& \lim _{n \rightarrow \infty} I_{n}[\beta ; f]=\lim _{n \rightarrow \infty} \sum_{i=1}^{n} w_{n i} f\left(x_{n i}\right)=\int_{a}^{b} f(x) d \beta(x), \\
& \lim _{n \rightarrow \infty} J_{n}[\beta ; f]=\lim _{n \rightarrow \infty} \sum_{i=1}^{n}\left|w_{n i}\right| f\left(x_{n i}\right)=\int_{a}^{b} f(x) d|\beta|(x) .
\end{aligned}
$$

Proof. This follows from Theorem 4.2 as $\tau(n, f)=\{1,2, \ldots, n\}$ for all $n \geqslant 1$, so $I_{n}^{*}[\beta ; f]=I_{n}[\beta ; f]$ and so on. Q.E.D.

For weight functions on the whole real line, there is the following corollary:

THEOREM 4.4. Let $P(x)$ be a polynomial, not vanishing identically. Let $\lambda \geqslant 1$ and $\gamma>-1$. Let $\alpha^{\prime}(x)$ be given by (3.27). Let $f: \mathbf{R} \rightarrow \mathbf{R}$ and $-\infty=y_{0}<y_{1}<y_{2}<$ $\cdots<y_{l+1}=\infty$ be such that $f$ is bounded and Riemann integrable in each compact subinterval of $\left(y_{i}, y_{i+1}\right), i=0,1,2, \ldots, l$. Further assume $P\left(\left|y_{i}\right|\right) \neq 0, i=1,2, \ldots, l$, and that $f^{2}(x) \alpha^{\prime}(x)$ is improperly Riemann integrable in $\mathbf{R}$, while

$$
\begin{gathered}
\lim _{x \rightarrow y_{i}}|f(x)|\left|x-y_{i}\right|^{1 / 2}|\log | x-y_{i}||=0, \quad i=1,2, \ldots, l . \\
\lim _{|x| \rightarrow \infty}|f(x)||P(x)|^{\gamma / 2} \exp \left(-|x|^{\lambda} / 2\right)|x|^{1 / 2}|\log | x||=0 .
\end{gathered}
$$

Let $k: \mathbf{R} \rightarrow \mathbf{R}$ be Lebesgue measurable, with

$$
\int_{-\infty}^{\infty} k^{2}(x)|P(|x|)|^{-\gamma} \exp \left(|x|^{\lambda}\right) d x<\infty
$$


Then, if $d \beta(x)=k(x) d x$,

$$
\lim _{n \rightarrow \infty} I_{n}^{*}[\beta ; f]=\int_{-\infty}^{\infty} f(x) k(x) d x, \quad \lim _{n \rightarrow \infty} J_{n}^{*}[\beta ; f]=\int_{-\infty}^{\infty} f(x)|k(x)| d x .
$$

If $l=0$, so that $f$ has no singularities interior to $\mathbf{R}$, we can replace $I_{n}^{*}$ and $J_{n}^{*}$ by $I_{n}$ and $J_{n}$, respectively.

Proof. This follows from Theorem 4.2 in the same way that Corollary 3.6 followed from Theorem 3.5. Q.E.D.

Remarks. (a) For the Hermite weight $\alpha^{\prime}(x)=\exp \left(-x^{2}\right)$, Smith, Sloan and Opie [14, Theorem 1] showed that (4.11) can be replaced by the weaker condition

$$
\int_{-\infty}^{\infty}\left\{|k(x)| \exp \left(x^{2} / 2\right)\right\}^{p} d x<\infty \text { some } p>1 .
$$

(For $p=2$ and the Hermite weight, this coincides with (4.11).) Their results were based on Nevai's results [9] on $L_{p}$ mean convergence of Lagrangian interpolation. No such results exist for the more general weight functions in Theorem 4.4, though if more were known about the orthogonal polynomials for these weight functions, such theorems could be proved. We note, however, that for the Hermite weight our condition $(4.10)$ reduces to

$$
\lim _{|x| \rightarrow \infty}|f(x)| \exp \left(-x^{2} / 2\right)|x|^{1 / 2}|\log | x||=0
$$

which is weaker than the condition on $f$ in Theorem 1 of [14]. Of course, as after Corollary 3.6, $|\log | x||$ can be replaced by $|\log | x||^{1 / 2+\varepsilon}$ or $|\log | x||^{1 / 2}|\log | \log |x|||^{1 / 2+\varepsilon}$ and so on, for any $\varepsilon>0$.

(b) Finally, we note that Theorem 5(ii) in Sloan and Smith [13] and Corollary 10.1.18 in Nevai [8, p. 181] show that for general weights, one cannot replace (4.6) in Theorem 4.2 by $\|d \beta / d \alpha\|_{\alpha, q}<\infty$, some $q>1$.

Acknowledgment. The authors would like to thank Professor P. Rabinowitz of the Weizmann Institute, Rehovot, Israel for pointing out [3] to them, and helping them to improve the presentation of the manuscript.

National Research Institute for Mathematical Sciences

C.S.I.R.

P. O. Box 395

Pretoria 0001, Republic of South Africa

Department of Computer Science

Techrion-Israel Institute of Technology

32000 Haifa, Israel

1. P. J. DaVIS \& P. Rabinowitz, "Ignoring the singularity in approximate integration," SIAM $J$. Numer. Anal., v. 2, 1965, pp. 367-383.

2. G. DE BARRA, Introduction to Measure Theor, Van Nostrand, New York, 1974.

3. H. ESSER, "Bemerkungen zu einem Satz von G. Freud über Quadraturkonvergenz," Computing, v. 8, 1971, pp. 216-220.

4. G. Freud, Orthogonal Polynomials, Pergamon Press, Budapest, 1971. (Translated by I. Földes.)

5. G. Freud, “On Markov-Bernstein-type inequalities and their applications," J. Approx. Theory, v. 19, 1977, pp. 22-37.

6. D. S. Lubinsky, "Gaussian quadrature, weights on the whole real line, and even entire functions with non-negative even order derivatives," J. Approx. Theory (To appear.)

7. D. S. Lubinsky \& P. Rabinowitz, "Rates of convergence of Gaussian quadrature for singular integrands," Math. Comp., v. 43, 1984, pp. 219-242. 
8. P. Nevai, “Orthogonal polynomials," Mem. Amer. Math. Soc., v. 18, 1979, No. 213.

9. P. NeVAi, "Mean convergence of Lagrange interpolation. II," J. Approx. Theory, v. 30, 1980, pp. 263-276.

10. P. Rabinowitz, "Gaussian integration in the presence of a singularity," SIAM J. Numer. Anal., v. 4, 1967, pp. 191-201.

11. P. Rabinowitz \& I. H. Sloan, "Product integration in the presence of a singularity," SIAM $J$. Numer. Anal., v. 21, 1984, pp. 149-166.

12. F. Riesz \& B. Sz. Nagy, Functional Analysis, Ungar, New York, 1955. (Translated by L. F. Boron.)

13. I. H. SloAN \& W. E. SMith, "Properties of interpolatory product integration rules," SIAM J. Numer. Anal., v. 19, 1982, pp. 427-442.

14. W. E. Smith, I. H. Sloan \& A. H. OpIE, "Product integration over infinite intervals I. Rules based on the zeros of Hermite polynomials," Math. Comp., v. 40, 1983, 519-535. 